\title{
Modeling Vibrational EELS: From Bulk to Point Defects
}

Guillaume Radtke ${ }^{1}$, Michele Lazzeri ${ }^{1}$, Dario Taverna ${ }^{1}$, Nicolas Menguy ${ }^{1}$, Fredrik Hage ${ }^{2}$, Demie Kepaptsoglou $^{3}$, Quentin Ramasse ${ }^{4}$, Ondrej Krivanek ${ }^{5}$ and Tracy Lovejoy ${ }^{5}$

${ }^{1}$ Sorbonne Université - CNRS, Paris, Ile-de-France, France, ${ }^{2}$ SuperSTEM Laboratory and University of Oxford, Daresbury, England, United Kingdom, ${ }^{3}$ SuperSTEM Laboratory and University of York, Daresbury, England, United Kingdom, ${ }^{4}$ SuperSTEM Laboratory and University of Leeds, Daresbury, England, United Kingdom, ${ }^{5}$ Nion Co., Kirkland, Washington, United States

Vibrational excitations are now accessible to high-resolution electron energy-loss spectroscopy (EELS) in a growing number of materials [1]. Most of the systems of interest in materials, earth or life sciences, however, display complex atomic structures whose dynamics can seldom be understood using empirical models or through simple comparisons with known references. A proper assessment of the origin of the different spectral features observed experimentally therefore relies both on first-principle calculations and on an accurate description of the scattering process [2].

We will first illustrate how density-functional theory (DFT) calculations provide invaluable insights into the dielectric properties of polar materials and, in particular, how they explain the orientation effects observed in aloof beam geometry experiments performed on anisotropic materials. Indeed, although the high-energy electron probe lacks the intrinsic angular selectivity of polarized photons used in optical spectroscopies, the scattering geometry in EELS provides a similar insight into the symmetry of vibrational excitations [3]. Figure 1 shows the result of two simple experiments performed on uniaxial $\mathrm{B}_{12} \mathrm{P}_{2}$ in which the electron beam and the surface are either parallel (a) or perpendicular (b) to the principal axis of symmetry of the crystal ( $c$ axis). The dramatic intensity variations of the peaks with the crystallographic orientation of the sample can be rationalized by identifying the symmetry of the modes. DFT calculations indeed reveal that modes generating an electric polarization in the plane perpendicular to the $c$ axis of the crystal ( $\mathrm{E}_{\mathrm{u}}$ symmetry) are enhanced in the first orientation while they are strongly reduced in the second. Conversely, modes generating an electric polarization along the $c$ axis $\left(\mathrm{A}_{2} \mathrm{u}\right.$ symmetry) display a weak intensity in the first orientation which increases in the second. EELS can therefore give access to the symmetry of vibrational modes as polarized IR spectroscopy does when carried out on single crystals.

In a second part, we will give an overview of the formalism employed when modelling experiments carried out in transmission geometry, a regime dominated by impact scattering, where a sizeable momentum is transferred to the specimen and phonon dispersions can be measured. In particular, we will show how DFT sheds light on the strong intensity variations observed between similar EELS active branches depending on whether they are recorded in the first or in higher Brillouin zones. In this context, the momentum dependence of nuclear charge screening by core and valence electrons will be discussed and illustrated by modelling E-Q data sets obtained recently in hexagonal BN [4].

Finally, a large part of this talk will be devoted to the modeling of atomic-resolution vibrational EELS acquired on point defects in graphene [5]. After discussing the general features of impurity-induced vibrational modes in solids and the insights EELS could provide in this field, we will present state-of-theart DFT calculations carried out on the particular case of a trivalent substitutional impurity of silicon. 
Results obtained from first-principles on supercells as large as 96 x 96 (18432 atoms) graphene unit cells -required to isolate the substitutional silicon atom- show how resonant modes result from the hybridization of local impurity modes with the continuum of "bulk" graphene. We will show how the projected phonon density of states mimics the dominant features of atomically-resolved dark-field EELS spectra.
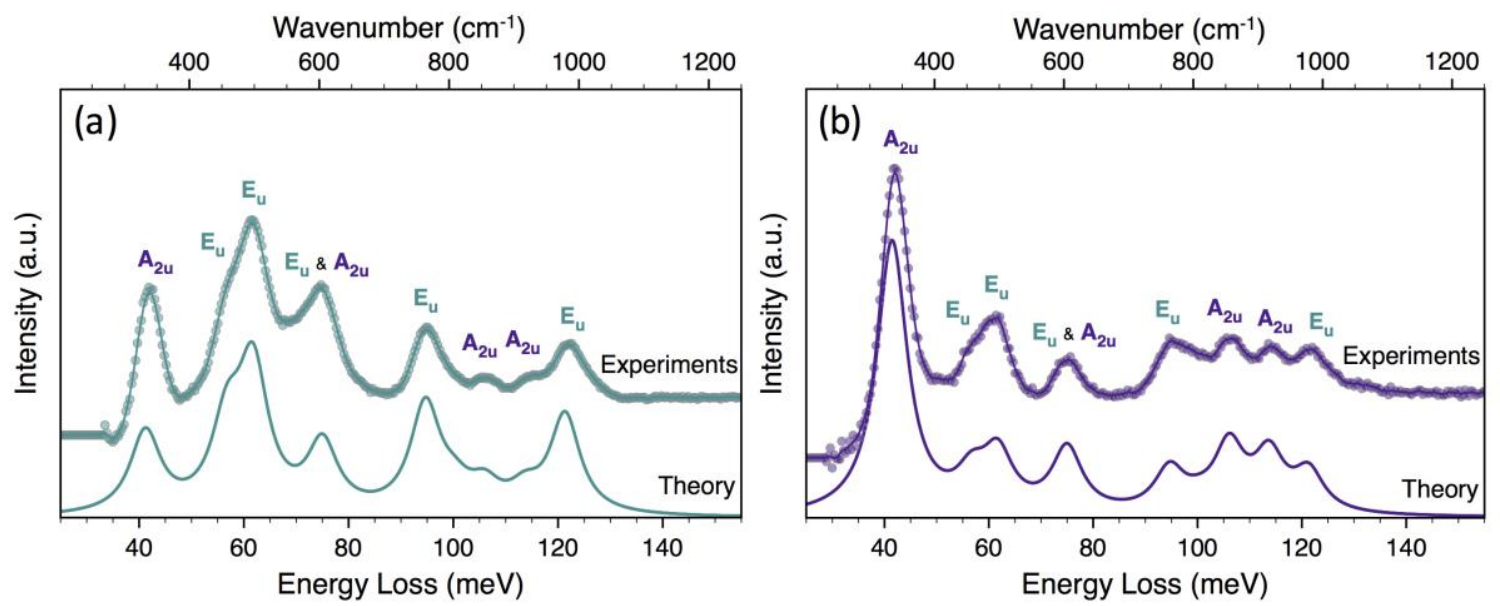

Figure 1. Experimental vibrational EELS spectra recorded on B12P2 in aloof geometry for two orientations of the crystals: (a) the crystal c axis is parallel to the surface and the electron beam; (b) the crystal $\mathrm{c}$ axis is perpendicular to the surface and to the electron beam trajectory. The theoretical spectra have been calculated in the framework of the local continuum dielectric model using a dielectric tensor calculated from first-principles.

References

[1] O.L. Krivanek, T. C. Lovejoy, N. Dellby, T. Aoki, R.W. Carpenter, P. Rez, E. Soignard, J. Zhu, P. E. Batson, M.J. Lagos, R.F. Egerton and P.A. Crozier, Nature 514 (2014) p.209.

[2] G. Radtke, D. Taverna, M. Lazzeri and E. Balan, Phys. Rev. Lett. 119 (2017) 027402.

[3] G. Radtke, D. Taverna, N. Menguy, S. Pandolfi, A. Courac, Y. Le Godec, O. L. Krivanek, and T. C. Lovejoy, Phys. Rev. Lett. 123 (2019) 256001.

[4] T.C. Lovejoy, G.J. Corbin, N. Dellby, N. Johnson, M.V. Hoffman, A. Mittelberger and O.L. Krivanek, Microsc. Microanal. 25 (Suppl 2) (2019) 628.

[5] F. S. Hage, G. Radtke, D. M. Kepaptsoglou, M. Lazzeri and Q. M. Ramasse, accepted in Science. 